\title{
THE INVERSE LIMIT OF SOME FREE ALGEBRAS
}

\author{
Deficated to the memory of Hanna Neumann
}

\author{
A. L. ALLEN and S. MORAN
}

(Received 12 October 1971)

Communicated by G. B. Preston

Let $\Omega\left[x_{1}, x_{2}, \cdots, x_{n}\right]$ denote the algebra of polynomials in variables $x_{1}, x_{2}, \cdots, x_{n}$ with coefficients from a fixed field $\Omega$ of characteristic zero, where $n=1,2, \cdots$. There exists a natural projection

$$
\phi_{n}: \Omega\left[x_{1}, x_{2}, \cdots, x_{n}\right] \rightarrow \Omega\left[x_{1}, x_{2}, \cdots, x_{n-1}\right]
$$

which maps $x_{n}$ onto 0 and all the other variables onto themselves, for $n=1,2, \cdots$. This enables one to construct the corresponding inverse limit

$$
\lim \Omega\left[x_{1}, x_{2}, \cdots, x_{n}\right] \text {, }
$$

which we here denote by $\Omega[x]$. The algebra $\Omega[x]$ has a natural degree function defined on it. We show

(1) $\Omega[x]$ has a countably generated subalgebra which is not isomorphic to a subalgebra of an algebra of polynomials in some set of variables over $\Omega$;

(2) the elements of finite degree in $\Omega[x]$ form a subalgebra of $\Omega[x]$ which is isomorphic to an algebra of polynomials in some set of variables over $\Omega$.

Let $I_{k}(n)$ denote the ideal of $\Omega\left[x_{1}, x_{2}, \cdots, x_{n}\right]$ consisting of all those polynomials whose homogeneous components of degree $<k$ are zero, for $k=2,3, \cdots$ and $n=1,2, \cdots$. Then the above defined $\phi_{n}$ induces a homomorphism

$$
\Omega\left[x_{1}, x_{2}, \cdots, x_{n}\right] / I_{k}(n) \rightarrow \Omega\left[x_{1}, x_{2}, \cdots, x_{n-1}\right] / I_{k}(n-1),
$$

for $n=2,3, \cdots$. This enables one to construct the corresponding inverse limit:

$$
\lim \left(\Omega\left[x_{1}, x_{2}, \cdots, x_{n}\right] / I_{k}(n)\right)
$$

for fixed $k=2,3, \cdots$. We show that this algebra is isomorphic to a subalgebra of an algebra of polynomials in some set of variables over $\Omega$ modulo the $k$-th power of the augmentation ideal of the latter algebra.

We have similar results for free nonassociative algebras with unit element 
over $\Omega$, free associative algebras with unit element over $\Omega$ and free Lie algebras over $\Omega$, where $\Omega$ can now be taken to be an arbitrary field. The case of free Lie algebras has already been considered by one of the authors [4]. Present considerations $^{(*)}$ show that [4] Theorem 2.4 is false. However the rest of the paper [4] is left undisturbed.

Finally we note that the above results hold if we consider the inverse limit of polynomial algebras in some arbitrary infinite collection of variables over $\Omega$ under the natural projections. The same can be said about the three other types of free algebras mentioned above. However for the sake of simplicity we shall restrict our attention to the countable case.

\section{Inverse limit of free Lie algebras}

Let $L_{n}$ denote the free Lie algebra on the free generators $x_{1}, x_{2}, \cdots, x_{n}$ with coefficients from the field $\Omega$, for $n=2,3, \cdots$. There exists a natural projection

$$
\phi_{n}: L_{n} \rightarrow L_{n-1} \text {, }
$$

which is defined by $\phi_{n}\left(x_{i}\right)=x_{i}$ for every $i<n$ and $\phi_{n}\left(x_{n}\right)=0$, for $n=2,3, \cdots$. Let $\boldsymbol{L}$ denote the corres-onding inverse limit

$$
\lim _{\leftarrow} L_{n} \text {. }
$$

$L$ contains a subalgebra naturally isomorphic to the free Lie algebra $L$ on the free generators $x_{1}, x_{2}, \cdots$, over $\Omega$. This is obtained by mapping the generator $x_{i}$ in $L$ onto the string of elements

$$
\left\langle 0, \cdots, 0, x_{i}, x_{i}, \cdots\right\rangle
$$

of $L$, where $x_{i}$ appears first in $i$ th place for $i=1,2, \cdots$. We shall identify $L$ with its image in $L$. Then $L$ can be considered to be the closure of $L$ in $L$ under the naturally defined topology in $L$. By $\Phi^{(n)}$ we denote the natural projection of $L$ onto $L_{n}$, which is cbtained by mapping an element of $L$ onto its $n$-th component for $n=1,2, \cdots$.

Note 1.1. For the rest of this section it is important to bear in mind that every subalgebra of a free Lie algebra over $\Omega$ is a free Lie algebra over $\Omega$ (Theorem of Širšov [5] and Witt [7]).

THEOREM 1.2. Let $L$ denote the above defined inverse limit of free Lie algebras over $\Omega$. Then $L$ has a subalgebra with a countably infinite number of generators which is not a free Lie algebra.

Proof. We shall show that any subalgebra of $L$ containing the elements

(*) We are grateful to Dr. D. W. Barnes for questioning the validity of [4] Lemma 2.1. 


$$
x_{i} \text { and } y_{i}=x_{i}+\left[x_{i+1}, x_{i}\right]+\cdots+\left[x_{i+j}, x_{i+j-1}, \cdots, x_{i}\right]+\cdots
$$

with $i=1,2, \cdots$ cannot be a free $L$ ie algebra. Suppose that to the contrary there exists some subalgebra of $\boldsymbol{L}$ which is a free Lie algebra and contains all these elements. As a free Lie algebra, this subalgebra has a natural grading. Hence a homogeneous element of some positive degree is a well defined notion in this subalgebra. The degree of a nonzero element of the subalgebra is defined to be the largest positive integer $d$ such that the homogeneous component of degree $d$ of this element is nonzero. Suppose that the degree of $y_{i}$ is $\beta_{i}$ for $i=1,2, \cdots$. Now

$$
y_{i}=x_{i}+\left[y_{i+1}, x_{i}\right]
$$

and induction on $r$ shows that

$$
y_{i}=x_{i}+\left[x_{i+1}, x_{i}\right]+\cdots+\left[x_{i+r-1}, x_{i+r-2}, \cdots, x_{i}\right]+\left[y_{i+r}, x_{i+r-1}, \cdots, x_{i}\right]
$$

for every $i=1,2, \cdots$ and $r=1,2, \cdots$. As the degree of each $x_{i}$ is greater than zero, we have that

$$
\beta_{i} \geqq \beta_{i+r}+r
$$

for every $i$ and $r$. Now take $r=\beta_{i}$. This gives that

$$
\beta_{i+B_{i}} \leqq 0
$$

which contradicts the fact that the degree of every nonzero element of a free Lie algebra is greater than zero. So every subalgebra of $\boldsymbol{L}$ which contains the elements

$$
x_{1}, x_{2}, \cdots, y_{1}, y_{2}, \cdots
$$

cannot be a free Lie algebra.

COROLlary 1.2.1. L is not a free Lie algebra.

We now turn to some positive results and start with the elementary

LEMMA 1.3. The inverse limit of the homogeneous subspaces of degree l of $\boldsymbol{L}_{n}$ under $\phi_{n}(n=2,3 \cdots)$ is a subspace $L_{(l)}$ of $L$ for $l=1,2, \cdots$. Every element of $L_{(l)}$ has a unique representation of the form

$$
\sum_{i=1}^{\infty} \alpha_{i l} b_{i}(l),
$$

where $\alpha_{i l} \in \Omega$ for all values of $i$ and $l$. The element $b_{i}(l)$ runs through all the basic monomials of degree $l$ on the free generators $x_{1}, x_{2}, \cdots$ of $L$ (for fixed $l$ ). In the unrestricted infinite sum $\Sigma^{*}$, the basic monomials of degree $l$ are ordered so that if

$$
\Phi^{(n)}\left(b_{i}(l)\right)=b_{i}(l) \text { while } \Phi^{(n)}\left(b_{j}(l)\right)=0
$$


for some positive integer $n$, then $b_{i}(l)$ appears before $b_{j}(l)$ in the unrestricted sum $\Sigma^{*}$.

Proof. $\boldsymbol{L}_{(i)}$ is obviously a subspace of $\boldsymbol{L}$. The above representation for an element of $\boldsymbol{L}_{(i)}$ follows from the corresponding representation for the homogeneous elements of degree $l$ in $L_{n}(n=1,2, \cdots)$. The uniqueness of the representation follows from the uniqueness of the representation in $L_{n}$ on applying $\Phi^{(n)}$ for a sufficiently large value of $n$.

NOTATION 1.4. For fixed $l$, the subspace $\Sigma_{m \geqq l} L_{(m)}$ is a subalgebra of $L$. Its closure in $\boldsymbol{L}$ will be denoted by ${ }_{(l)} \boldsymbol{L}$. It is not difficult to see that ${ }_{(l)} \boldsymbol{L}$ is the closure of ${ }^{l} \boldsymbol{L}$ in $\boldsymbol{L}$, where ${ }^{l} \boldsymbol{L}$ is $\boldsymbol{l}$ th member of the lower central series of $\boldsymbol{L}$.

For the sake of completeness we repeat Construction 2.2 and a more detailed version of Lemma 2.3 of Moran [4].

Construction 1.5. Since ${ }_{i} L /{ }_{i+1} L$ is a vector space over $\Omega$, it is possible to construct a set $O_{i}$ for every $i$. Let $O_{1}=N_{1}$ be a set of elements of $\boldsymbol{L}$ that is linearly independent modulo ${ }_{2} L$. Suppose that the sets $O_{v}$ and $N_{v}$ have already been defined for all $v<n$ (where $n>1)$ and the elements of the sets $O_{v}(v=1,2, \cdots, n-1)$ have been ordered so that an element of $O_{v}$ is greater than an element of $O_{v}$ if $v>v^{\prime}$. We define $N_{n}$ to be the set of all basic monomials on the elements of the sets $O_{1}, O_{2}, \cdots, O_{n-1}$ which belong to ${ }_{n} L$ but do not belong to ${ }_{n+1} L$. Finally $O_{n}$ is a set of elements of ${ }_{n} L$ which is linearly independent modulo the subalgebra generated by ${ }_{n+1} L$ and $N_{n}$.

Lemma 1.6. Let $O_{1}, O_{2}, \cdots, O_{n-1}$ be finite sets. Then

(a) $N_{n}$ is a set of linearly independent elements of ${ }_{n} \boldsymbol{L}$ modulo ${ }_{n+1} \boldsymbol{L}$ and

(b) $\Phi^{(p)}\left(N_{n}\right)$ is a set of linearly independent elements of ${ }^{n} L_{p}$ modulo ${ }^{n+1} L_{n}$, for all sufficiently large values of $p$, for $n=1,2, \cdots$.

Proof. (a) We proceed by induction on $n$. The result is true, by construction, when $n=1$. Suppose that the result is true for $N, N_{2}, \cdots, N_{n-1}$. Now as these sets are finite, for every $m$, where $1 \leqq m \leqq n-1$, there exist elements $d_{i}(m)$ of ${ }_{m} L$ and positive integers $p(m)$ and $q(m)$ such that

$$
{ }_{m} L / m+1=\left(\sum_{l=1}^{q(m)}\left\{d_{i}(m)+{ }_{m+1} L\right\}\right) \oplus\left(\sum_{i>p(m)}^{*}\left\{b_{i}(m)+{ }_{m+1} L\right\}\right)
$$

and

$$
\left(N_{m} \cup O_{m}\right)+{ }_{m+1} L \subseteq \sum_{i=1}^{q(m)}\left(\left\{d_{i}(m)+{ }_{m+1} L\right\}\right) .
$$

Here $\Sigma$ and $\Sigma^{*}$ denote restricted and unrestricted direct sums respectively, while $\Sigma_{i>p(m)}^{*}$ is to mean that those and only those basic monomials of weight $m$ on $x_{1}, x_{2}, \cdots$ occur in the unrestricted direct sum which satisfy the condition 


$$
\Phi^{(p(m)+1)}\left(b_{i}(m)\right)=0 .
$$

Suppose that contrary to our lemma, the elements of $N_{n}$ are linearly dependent modulo $_{n+1} L$, then there exist scalars (not all zero) such that

$$
c=\gamma_{n_{1}} c_{n_{1}}+\cdots+\gamma_{n_{k}} c_{n_{k}} \text { belongs to }{ }_{n+1} L,
$$

where every $c_{n_{i}}$ is an element of $N_{n}$. Let $p$ denote the maximum of $p(1), p(2), \cdots, p(n-1)$ and apply the homomorphism $\Phi^{(p)}$ to $c$. Then

$$
\Phi^{(p)}(c)=\gamma_{n_{1}} \Phi^{(p)}\left(c_{n_{1}}\right)+\cdots+\gamma_{n_{k}} \Phi^{(p)}\left(c_{n_{k}}\right) \text { belongs to }{ }^{n+1} L_{p} .
$$

This implies that for some $l(\leqq n-1)$

$$
\Phi^{(p)}\left(N_{l}\right) \cup \Phi^{(p)}\left(O_{l}\right)
$$

must be a set of linearly dependent elements of ${ }_{l} L$ modulo ${ }_{l+1} L$. Otherwise, by a Theorem of Širšov [5], the elements of the set

$$
\Phi^{(p)}\left(O_{1}\right) \cup \Phi^{(p)}\left(O_{2}\right) \cup \cdots \cup \Phi^{(p)}\left(O_{n-1}\right)
$$

freely generate a free Lie subalgebra of $L_{p}$. This contradicts the fact that $\Phi^{(p)}(c)$ belongs to ${ }^{n+1} L_{p}$. Hence there exist scalars $\varepsilon_{l i}, \varepsilon_{l i}^{\prime}$ (not all zero) such that

$$
\varepsilon_{l 1} \Phi^{(p)}\left(a_{l 1}\right)+\cdots+\varepsilon_{l k} \Phi^{(p)}\left(a_{l k}\right)+\varepsilon_{l 1}^{\prime} \Phi^{(p)}\left(c_{l 1}\right)+\cdots+\varepsilon_{l k^{\prime}}^{\prime} \Phi^{(p)}\left(c_{i k^{\prime}}\right)
$$

belongs to ${ }_{l+1} L$, where every $a_{l i} \in O_{l}$ and every $c_{l i} \in N_{l}$. Thus the element

$$
\bar{a}_{l}=\varepsilon_{l 1} a_{l 1}+\cdots+\varepsilon_{l k} a_{l k}+\varepsilon_{l 1}^{\prime} c_{l 1}+\cdots+\varepsilon_{l k^{\prime}}^{\prime} c_{l k},
$$

has the following properties:

(1) it does not belong to ${ }_{l+1} L$, by the induction hypothesis and Construction 1.5 ;

(2) it belongs to $\sum_{i>p}^{*}\left(\left\{b_{i}(l)+{ }_{l+1} L\right\}\right)$ modulo ${ }_{l+1} L$;

(3) it belongs to $\sum_{i}\left(\left\{d_{i}(l)+{ }_{l+1} L\right\}\right)$ modulo ${ }_{l+1} L$.

The above three properties of the element $\bar{a}_{l}+{ }_{l+1} L$ contradict the direct decomposition (1.6.1) for $l_{l} L / t+1$. This establishes part (a) of our lemma.

(b) We proceed by induction on $n$ as in part (a) and use the direct decomposition (1.6.1) for $m=1,2, \cdots, n$ together with Lemma 1.6(a). In fact suppose that

$$
\gamma_{n_{1}} \Phi^{(p(n))}\left(c_{n_{1}}\right)+\cdots+\gamma_{n_{k}} \Phi^{(p(n))}\left(c_{n_{k}}\right) \text { belongs to }{ }^{n+1} L_{p(n)} .
$$

Then the element (where we are using the same notation as in (a))

$$
c=\gamma_{n_{1}} c_{n_{1}}+\cdots+\gamma_{n_{k}} c_{n_{k}}
$$

modulo ${ }_{n+1} L$ belongs to

$$
\sum_{i>p(n)}^{*}\left(\left\{b_{i}(n)+{ }_{n+1} L\right\}\right) \text { and } \sum_{i=1}^{q(n)}\left(\left\{d_{i}(n)+{ }_{n+1} L\right\}\right) .
$$


This implies that $c$ belongs to ${ }_{n+1} L$, which contradicts Lemma 1.6(a). Hence the required result (b) follows.

THEOREM 1.7. Let $O_{1}, \cdots, O_{l}$ be subsets of $L$ which are constructed as in Construction 1.5. Then the elements of the set $O_{1} \cup \cdots \cup O_{1}$ freely generate a free Lie algebra over $\Omega$.

Proof. It follows from Lemma 1.6(a) that the corresponding set $N_{n}$ is linearly independent modulo ${ }_{n+1} L$, for every $n$. Hence the elements of the set

$$
O_{1} \cup O_{2} \cup \cdots \cup O_{l}
$$

form a set of free generators for a free Lie algebra over $\Omega$.

This gives rise immediately to the following

THEOREM 1.7.1. Every finitely generated subalgebra of $L$ is a free Lie algebra over $\Omega$.

NOTE 1.8. We now turn to a consideration of the subalgebra $L^{*}$ of $L$ which consists of all elements of finite degree together with the zero element. An element of $L$ is said to have degree $m$ if and only if it belongs to $\sum_{n \leqq m} L_{(n)}$ but not to $\Sigma_{n<m} L_{(n)}$. Hence

$$
L^{*}=\sum_{n=1}^{\infty} L_{(n)}
$$

THEOREM 1.9. The subalgebra $L^{*}$ of $L$ consisting of all elements of finite degree is a free Lie algebra over $\Omega$.

Proof. Let $O_{1}, O_{2}, \cdots, O_{n}, \cdots$ be maximal sets satisfying the conditions of Construction 1.5 and also the conditions $O_{n} \subset L_{(n)}$ for $n=1,2, \cdots$. Then $\bigcup_{n=1}^{\infty} O_{n}$ is a set of generators for $L^{*}$. The elements of the set $N_{n}$ are linearly independent modulo ${ }_{n+1} L$, for $n=1,2, \cdots$. For, by Lemma 1.6(a), every finite subset of $N_{n}$ is linearly independent modulo ${ }_{n+1} L$. Hence the set $\bigcup_{n=1}^{\infty} O_{n}$ is a set of free generators for $L^{*}$.

We now quickly outline the corresponding result in the nilpotent case.

THEOREM 1.10. Let $\overline{{ }^{k} L}$ denote the closure of $k$-th member of the lower central series of $\boldsymbol{L}$ in $L$. Then $\boldsymbol{L}^{\bar{k} \boldsymbol{L}}$ is isomorphic to a subalgebra of a free $(k-1)$-th nilpotent Lie algebra over $\Omega$.

Proof. By Lemma 1.3 and Notation 1.4, every elements of $L /{ }^{\bar{k} L}$ has a unique representation of the form

$$
\sum_{i=1}^{k-1}\left(\sum_{i=1}^{\infty} \alpha_{i l} b_{i}(l)\right)
$$


modulo $\overline{\boldsymbol{L}}$. Let $O_{1}, \cdots, O_{k-1}$ be maximal subsets of $\boldsymbol{L}$ satisfying the conditions of Construction 1.5 and such that $O_{n} \subset L_{(n)}$ for $n=1,2, \cdots, k-1$. Then $O_{1} \cup \cdots \cup O_{k-1}$ is a set of generators for $\boldsymbol{L}$ modulo ${ }^{k} \boldsymbol{L}$. The fact that $\boldsymbol{L} /^{\bar{L} \boldsymbol{L}}$ is isomorphic to a subalgebra of a free $(k-1)$ th Lie algebra over $\Omega$ is now proved as follows. One takes a free $(k-1)$ th nilpotent Lie algebra over $\Omega$ on free generators $y_{\alpha(i)}, \alpha(i) \in M(i)$, with $|M(i)|=\left|O_{i}\right|$ for $i=1,2, \cdots, k-1$. The isomorphism is defined by mapping the distinct elements of $O_{i}$ onto distinct simple basic monomials with the last component being the least $y_{\alpha}$ of weight $i$ in the generators $y_{\alpha(i)}, \alpha(i) \in M(i)$, for $i=1,2, \cdots, k-1$. The mapping defined in this way is obviously one-to-one and single-valued, by Lemma 1.6(a). The fact that it preserves Lie products follows from Hall's Collecting Process.

NoTe 1.10.1. It is not difficult to verify that $L / \bar{k} \boldsymbol{L}$ is isomorphic to $\lim \left(L_{n} /{ }^{k} L_{n}\right)$ under the homomorphisms of $L_{n} /{ }^{k} L_{n} \rightarrow L_{n-1} /{ }^{k} L_{n-1}$ induced by $\phi_{n}: L_{n}^{\leftarrow} \rightarrow L_{n-1}$, for $n=2,3, \cdots$.

\section{Inverse limit of free associative algebras}

Let $A_{n}$ denote the free associative algebra with unit element on the free generators $x_{1}, x_{2}, \cdots, x_{n}$ with coefficients from the field $\Omega$, for $n=1,2, \cdots$. There exists a natural projection

$$
\phi_{n}: A_{n} \rightarrow A_{n-1}
$$

which is defined by $\phi_{n}\left(x_{i}\right)=x_{i}$ for $i<n$ and $\phi_{n}\left(x_{n}\right)=0$, for $n=2,3, \cdots$. Let $A$ denote the corresponding inverse limit

$$
\lim A_{n} \text {. }
$$

$A$ contains a subalgebra naturally isomorphic to the free associative algebra $A$ with unit element on free generators $x_{1}, x_{2}, \cdots$ over $\Omega$. This is obtained by mapping the variable $x_{i}$ in $A$ onto the string of elements

$$
\left\langle 0, \cdots, 0, x_{i}, x_{i}, \cdots\right\rangle
$$

of $A$, where $x_{i}$ appears first in $i$ th place, for $i=1,2, \cdots$. We shall identify $A$ with its image in $\boldsymbol{A}$. Then $\boldsymbol{A}$ can be considered to be the closure of $A$ in $\boldsymbol{A}$ under the naturally defined topology in $A$. By $\Phi^{(n)}$ we denote the natural projection of $\boldsymbol{A}$ onto $A_{n}$, which is defined by mapping an element of $A$ onto its $n$th component, for $n=1,2, \cdots$.

NoTE 2.1. In this section it is important to remember that not every subalgebra (with unit element) of a free associative algebra with unit element over $\Omega$ is a free associative algebra with unit element over $\Omega$. The simplest counterexample is given by the subalgebra with unit element generated by $x^{2}$ and $x^{3}$ in $\Omega[x]$. 
THEOREM 2.2. Let $\boldsymbol{A}$ denote the above defined inverse limit of free associative algebras with unit element over $\Omega$. Then $A$ has a subalgebra with unit element which has a countably infinite number of generators but is not isomorphic to a subalgebra of a free associative algebra (with unit element) over $\Omega$.

Proof. We shall show that any subalgebra with unit element of $\boldsymbol{A}$ which contains the elements

$$
x_{i} \text { and } y_{i}=x_{i}+{ }_{x} x_{i+1} x_{i}+\cdots+x_{i+j} \cdot x_{i+j-i} \cdots x_{i}+\cdots
$$

with $i=1,2, \cdots$ cannot be contained in a free associative algebra with unit element over $\Omega$. Suppose that to the contrary there exists some subalgebra with unit element of $\boldsymbol{A}$ which can be isomorphically embedded in some free associative algebra with unit element over $\Omega$ and contains all these elements $x_{i}, y_{i}$. Then this subalgebra inherits a degree function from the free associative algebra with unit element in which it is embedded. Suppose that the degree of $y_{i}$ is $\beta_{i}$ for $i=1,2, \cdots$. Now

$$
y_{i}=x_{i}+y_{i+1} \cdot x_{i}
$$

and induction on $r$ shows that

$$
y_{i}=x_{i}+x_{i+1} \cdot x_{i}+\cdots+x_{i+r-1} \cdot x_{i+r-2} \cdots x_{i}+y_{i+r} \cdot x_{i+r-1} \cdots x_{i}
$$

for every $i=1,2, \cdots$ and $r=1,2, \cdots$. As the degree of each $x_{i} \geqq 1$, we have that

$$
\beta_{i} \geqq \beta_{i+r}+r \text { for every } i \text { and } r \geqq 1 \text {. }
$$

Take $r=\beta_{i}$. This gives that

$$
\beta_{i+} \beta_{i} \leqq 0
$$

which contradicts the fact that every $y_{i}$ is not a scalar from $\Omega$. So every subalgebra with unit element of $\boldsymbol{A}$ which contains the elements

$$
x_{1}, x_{2}, \cdots, y_{1}, y_{2}, \cdots
$$

cannot be isomorphic to a subalgebra with unit element of a free associative algebra with unit element over $\Omega$.

Corollary 2.2.1. A is not isomorphic to a subalgebra with unit element of a free associative algebra with unit element over $\Omega$.

As before we have a result of the following form:

LEMMA 2.3. The inverse limit of the homogeneous subspaces of degree $l$ of $A_{n}$ under $\phi_{n}(n=1,2, \cdots)$ is a subspace $\boldsymbol{A}_{(l)}$ of $\boldsymbol{A}$, for $l=1,2, \cdots$. Every element of $\boldsymbol{A}_{(l)}$ has a unique representation of the form 


$$
\sum_{i=1}^{\infty} \alpha_{i l} \cdot m_{i}(l)
$$

where $\alpha_{i l} \in \Omega$ for all values of $i$ and $l$. Here $m_{i}(l)$ runs through all the associative monomials of degree $l$ on the free generators $x_{1}, x_{2}, \cdots$ (for fixed $l$ ) of $A$. In the unrestricted direct sum $\Sigma^{*}$ the monomials of degree $l$ are ordered so that if

$$
\Phi^{(n)}\left(m_{i}(l)\right)=m_{i}(l) \text { while } \Phi^{(n)}\left(m_{j}(l)\right)=0
$$

for some positive integer $n$, then $m_{i}(l)$ appears before $m_{j}(l)$ in the unrestricted sum $\Sigma^{*}$.

Notation 2.4. $\sum_{i \geqq l} A_{(i)}$ is a subalgebra of $A$. Its closure in $A$ will be denoted by ${ }_{l} A$. It is not difficult to see that ${ }_{l} A$ is the closure of $\left({ }_{1} A\right)^{l}$ in $A$, where ${ }_{1} A$ is the augmentation ideal of $A$ and $\left({ }_{1} A\right)^{l}$ is the ideal of ${ }_{1} A$ generated by all finite products of more than $(l-1)$ elements in ${ }_{1} A$.

Construction 2.5. Since ${ }_{i} \boldsymbol{A} /{ }_{i+1} \boldsymbol{A}$ is a vector space over $\Omega$, it is possible to construct the following sets $O_{i}$. Let $O_{1}=N_{1}$ be a set of elements of ${ }_{1} A$ that is linearly independent modulo ${ }_{2} A$. Suppose that the sets $O_{v}$ and $N_{v}$ have already been defined for all $v<n$ (where $n \geqq 1$ ) and the elements of the sets $O_{v}$ $(v=1,2, \cdots, n-1)$ have been ordered so that an element of $O_{v}$ is greater than an element of $O_{v}$ if $v>v^{\prime}$. We define $N_{n}$ to be the set of all monomials on the elements of the set $O_{1} \cup O_{2} \cup \ldots \cup O_{n-1}$ which belong to ${ }_{n} A$ but do not belong to ${ }_{n+1} A$. Finally $O_{n}$ is a set of elements of ${ }_{n} A$ which is linearly independent modulo the subalgebra generated by ${ }_{n+1} A$ and $N_{n}$.

In order to establish a result similar to Lemma 1.6 , we have to introduce a new concept.

Definition 2.6. Let $x$ be a nonzero element of ${ }_{1} \boldsymbol{A}$. Then $x$ belongs to ${ }_{1} \boldsymbol{A}$ but not to $l_{l+1} A$, for some positive integer $l$. By Lemma 2.3, the element $x$ will have a unique representation of the form

$$
\sum_{i=1}^{\infty} f_{i}(l-1) \cdot x_{i}
$$

where $f_{i}(l-1)$ is an element of ${ }_{l-1} A$, for $i=1,2, \cdots$. The element $x$ belonging to a subalgebra $B$ (with unit element) of $A$ is said to be right stable in $B$ if and only if $f_{i}(l-1)$ belongs to $B$ modulo ${ }_{l} A$, for $i=1,2, \cdots$.

LEMMA 2.7. Let $\mathrm{O}_{1}, \mathrm{O}_{2}, \cdots, \mathrm{O}_{n}, \cdots$ be sets of elements in $\mathrm{A}$ defined as in Construction 2.5. Suppose also that $\cup O_{n}$ is a right stable set of elements in the subalgebra (with unit element) generated by the elements of $\cup O_{n}$ in $\boldsymbol{A}$. Then $\cup O_{n}$ freely generates a free associative algebra with unit element over $\mathbf{\Omega}$ in $\boldsymbol{A}$. 
Proor. It is sufficient to show that if $o_{1}, o_{2}, \cdots, o_{k}$ are distinct elements of $\cup O_{n}$ with $o_{j} \in \in_{\alpha j} A$ but $o_{j} \notin_{\alpha_{j}+1} A$ for $j=1,2, \cdots, k$ and $f_{i}(o)$ is an associative polynomial on the elements of $\cup O_{n}$ with $f_{j}(o) \in_{\beta_{j}} A$ but $f_{j}(o) \notin_{\beta_{j}+1} A$ for $j=1,2, \cdots, k$ with ${ }^{\dagger}$

for $j=1,2, \cdots, k$, then

$$
\alpha_{j}+\beta_{j}=n
$$

$$
\sum_{j=1}^{k} o_{j} \cdot f_{j}(o) \text { does not belong to }{ }_{n+1} A .
$$

Suppose that this latter result does not hold. Then

$$
\sum_{j=1}^{k} o_{j} \cdot f_{j}(o) \text { belongs to }{ }_{n+1} A \text {. }
$$

Every $\beta_{j} \geqq 1$, otherwise this would contradict the construction of the set $O_{n}$. Since $\cup O_{n}$ is right stable, we have that

$$
\sum_{j=1}^{k} o_{j} \sum_{i=1}^{\infty} f_{j i}(o) \cdot x_{i} \text { belongs to }{ }_{n+1} A,
$$

where $f_{j i}(o)$ is an associative polynomial in the elements of $\cup O_{n}$ which belongs to $\beta_{i}-1$ A for $j=1,2, \cdots, k$ and $i=1,2, \cdots$. Now, by the uniqueness of the representation as explained in Definition 2.6, we have that

$$
\sum_{j=1}^{k} o_{j} \cdot f_{j i}(o) \text { belongs to }{ }_{n} A \text {. }
$$

Induction on $n$ and Construction 2.5 now completes the proof. The case $n=1$, follows at once from the construction of the set $O_{1}$.

NoTE 2.7.1. There exists a similar result to the above emma when right stable is replaced by left stable.

Note 2.8. We now consider the subalgebra $\boldsymbol{A}^{*}$ with unit element which consists of all elements of finite degree in $A$. An element of $A$ is said to have degree $m$ if and only if it belongs to $\Sigma_{n \leqq m} A_{(n)}$ but does not belong to $\Sigma_{n<m} A_{(n)}$. Hence

$$
A^{*}=\Omega+\sum_{n=1}^{\infty} A_{(n)} .
$$

THEOREM 2.9. The subalgebra $\boldsymbol{A}^{*}$ of $\boldsymbol{A}$ which consists of all elements of finite degree is a free associative algebra with unit element over $\Omega$.

Proof. Let $O_{1}, O_{2}, \cdots, O_{n}, \cdots$ be maximal subsets of $A^{*}$ satisfying the conditions of Construction 2.5 and also the conditions $O_{n} \subset \boldsymbol{A}_{(n)}$ for $n=1,2, \cdots$.

\footnotetext{
${ }^{\dagger}$ By induction we may assume that all the $\beta_{i}$ are calculated in the natural way.
} 
Then it follows at once that $\cup O_{n}$ is a right stable set of generators for $A^{*}$. The required result now follows from Lemma 2.4.

We now consider the nilpotent case.

THEOREM 2.10. Let ${ }_{1} \overline{A^{k}}$ denote the closure of the kth power of the augmentation ideal ${ }_{1} \boldsymbol{A}$ in $\boldsymbol{A}$. Then $\boldsymbol{A} /{ }_{1} \overline{A^{k}}$ is isomorphic to a subalgebra with unit element of a free $(k-1)$ th nilpotent associative algebra with unit element over $\Omega$.

Proof. By Lemma 2.3 and Notation 2.4, every element of ${ }_{1} A /{ }_{1} \bar{A}^{k}$ has a unique representation of the form

$$
\sum_{l=1}^{k-1}\left(\sum_{i=1}^{\infty} \alpha_{i l} \cdot m_{i}(l)\right)
$$

modulo ${ }_{1} \overline{A^{k}}={ }_{k} A$. Let $O_{1}, O_{2}, \cdots, O_{k-1}$ be maximal subsets of $A$ satisfying the conditions of Construction 2.5 and such that $O_{n} \subset A_{(n)}$ for $n=1,2, \cdots, k-1$. Then $O_{1} \cup O_{2} \cup \cdots \cup O_{k-1}$ is a set of generators for $A$ modulo ${ }_{k} A$. The fact that $\boldsymbol{A} /{ }_{1} \overline{\boldsymbol{A}}^{k}$ is isomorphic to a subalgebra with unit element of a free $(k-1)$ th nilpotent associative algebra with unit element over $\Omega$ is shown as follows. One takes a free $(k-1)$ th nilpotent associative algebra with unit element over $\Omega$ on free generators $y_{\alpha(i)}, \alpha(i) \in M(i)$, with $|M(i)|=\left|O_{i}\right|$, for $i=1,2, \cdots, k-1$. The appropriate isomorphism is defined by mapping an element of $O_{i}$ onto the corresponding $y_{\alpha(i)}^{i}$, for $i=1,2, \cdots, k-1$. The above defined mapping is one-to-one and single-valued by Lemma 2.7. It obviously preserves sums and products.

Nore 2.10.1. It is not difficult to verify that $\boldsymbol{A} /{ }_{k} \boldsymbol{A}$ is isomorphic to $\lim \left(A_{n} /{ }_{1} A_{n}^{k}\right)$ under the homomorphisms of $A_{n} / A_{1} A_{n}^{k}$ onto $A_{n-1} / A_{n-1}^{k}$ induced by $\overline{\bar{\phi}}_{n}: A_{n} \rightarrow A_{n-1}$ for $n=2,3, \cdots$.

\section{Inverse limit of polynomial algebras}

Let $C=\Omega\left[x_{1}, x_{2}, \cdots, x_{n}\right]$ be the usual algebra of polynomials in the variables $x_{1}, x_{2}, \cdots, x_{n}$ with coefficients from the field $\Omega$, for $n=1,2, \cdots$. There exists a natural projection

$$
\phi_{n}: C_{n} \rightarrow C_{n-1}
$$

which is defined by $\phi_{n}\left(x_{i}\right)=x_{i}$ for $i<n$ and $\phi_{n}\left(x_{n}\right)=x_{n}$, for $n=2,3, \cdots$. Let $C$ denote the corresponding inverse limit

$$
\lim _{\leftarrow} C_{n} \text {. }
$$

C contains a subalgebra naturally isomorphic to $C=\Omega\left[x_{1}, x_{2}, \cdots, x_{n}, \cdots\right]$. We shall identify $C$ with its image in $C$. Then $C$ is the closure of $C$ in $C$ under the naturally defined topology in $C$. 
NoTE 3.1. Every subalgebra with unit element of $\Omega\left[x_{\alpha} ; \alpha \in M\right]$ is not of the same form. The counter example given in Note 2.1 works also here.

THEOREM 3.2. Let $\boldsymbol{C}$ denote the above defined inverse limit of polynomial algebras with unit element over $\Omega$. Then $C$ has a subalgebra with unit element which has a countably infinite number of generators but is not isomorphic to a subalgebra with unit element of an algebra of polynomials in some set of variables over $\Omega$.

Proof. The proof given for Theorem 2.2 works also here.

COROLlary 3.2.1. The algebra $\mathrm{C}$ is not isomorphic to a subalgebra with unit element of an algebra of polynomials in some set of variables over $\Omega$.

As before we have a result of the following form:

Lemma 3.3. The inverse limit of the homogeneous subspaces of degree $l$ of $C_{n}$ under $\phi_{n}(n=1,2, \cdots)$ is a subspace $\boldsymbol{C}_{(l)}$ of $\boldsymbol{C}$, for $l=1,2, \cdots$. Every element of $C_{(l)}$ has a unique representation of the form

$$
\sum_{i=1}^{\infty} \alpha_{i l}, m_{i}(l),
$$

where $\alpha_{i l} \in \mathbf{\Omega}$ for all values of $i$ and $l$. Here $m_{i}(l)$ runs through all the monomials of degree lon the free generators $x_{1}, x_{2}, \cdots,($ for fixed $l)$ of $C$. In the unrestricted direct sum $\Sigma^{*}$ the monomials of degree l are ordered so that if $m_{j}(l)$ contains one of the variables

$$
x_{n+1}, x_{n+2}, \cdots
$$

but $m_{i}(l)$ does not, then $m_{i}(l)$ appears before $m_{j}(l)$ in the unrestricted direct sum $\Sigma^{*}$.

Notation 3.4. $\Sigma_{i \geqq l} C_{(i)}$ is a subalgebra of $C$. Its closure in $C$ will be denoted by ${ }_{l} C$. It is not difficult to see that ${ }_{l} C$ is the closure of $\left({ }_{1} C\right)^{l}$ in $C$, where ${ }_{1} C$ is the augmentation ideal of $C$ and $\left({ }_{1} C\right)^{l}$ is the ideal of ${ }_{1} C$ generated by all finite products of more than $(l-1)$ elements in ${ }_{1} C$.

Construction 3.5. We construct the sets $O_{i}$ and $N_{i}$ for $i=1,2, \cdots$ in the algebra $C$ in the same way as they were constructed in Construction 2.5 for the associative algebra $\boldsymbol{A}$.

We now have the useful

Lemma 3.6. Let $O_{1}, O_{2}, \cdots, O_{n}$ be finite subsets of $C_{(1)}, C_{(2)}, \cdots, C_{(n)}$ respectively constructed according to Construction 3.5. The set of elements of $O_{n} \cup N_{n}$ is linearly independent over $\Omega$ if and only if $\dagger$ if $\Phi^{(s)}\left(O_{n} \cup N_{n}\right)$ is linearly independent over $\Omega$ for all sufficiently large values of $s$.

$\dagger \Phi^{(s)}$ denotes the natural projection of $C$ onto $C_{s}$. 
Proof. By Fuchs [1] Theorem 47.1 proof, we have that

and

$$
C_{(n)}=\sum_{i=1}^{q(n)}\left\{d_{i}(n)\right\}+\sum_{i \geqq s(n)}^{*}\left\{m_{i}(n)\right\}
$$

$$
O_{n} \cup N_{n} \subset \sum_{i=1}^{q(n)}\left\{d_{i}(n)\right\}
$$

where, using the notation of Lemma 3.3, every monomial appearing as generator in the unrestricted direct sum $\Sigma^{*}$ involves at least one of the variables

$$
x_{s(n)}, x_{s(n)+1}, \cdots,
$$

and all such monomials occur. Assume firstly that $O_{n} \cup N_{n}$ is linearly independent over $\Omega$. Suppose that

$$
\alpha_{1} \Phi^{(s)}\left(z_{1}\right)+\cdots+\alpha_{r} \Phi^{(s)}\left(z_{r}\right)=0,
$$

where $\alpha_{1}, \cdots, \alpha_{r}$ are scalars from $\Omega$, every $z_{i}$ belongs to $O_{n} \cup N_{n}$ and $s$ is an integer not less than $s(n)$. By the above direct decomposition, we have that

$$
\alpha_{1} z_{1}+\cdots+\alpha_{r} z_{r}=0 .
$$

This implies that every $\alpha_{i}=0$, that is, $\Phi^{(s)}\left(O_{n} \cup N_{n}\right)$ is linearly independent over $\Omega$. Conversely it follows at once that if $\Phi^{(s)}\left(O_{n} \cup N_{n}\right)$ is linearly independent over $\Omega$, then $O_{n} \cup N_{n}$ cannot be linearly dependent over $\Omega$.

Note 3.7. We now consider the subalgebra $C^{*}$ with unit element which consists of all elements of finite degree in $C$. An element of $C$ is said to have degree $m$ if and only if it belongs to $\Sigma_{n \leqq m} C_{(n)}$ but not to $\Sigma_{n<m} C_{(n)}$. Hence

$$
C^{*}=\Omega+\sum_{n=1}^{\infty} C_{(n)} \text {. }
$$

THEOREM 3.8. Let $\Omega$ be a field of characteristic zero. Then the subalgebra $C^{*}$ of elements of finite degree in $\boldsymbol{C}$ is an algebra of polynomials in some set of variables (taken from $C$ ) over $\Omega$.

Proof. Let $O_{1}, O_{2}, \cdots, O_{n}, \cdots$ be maximal subsets of $C^{*}$ satisfying the conditions given in Coustruction 3.5 and such that $O_{n} \subset C_{(n)}$ for every $n$. Then it follows at once from the maximality condition that $\cup O_{n}$ is a set of generators for $C^{*}$. We shall now prove by induction on $n$ that the elements of $N_{n}$ are linearly independent over $\Omega$. This result is trivially true by construction for $n=1$. Suppose that it is true for $N_{m}$, whenever $m<n$.

Suppose that contrary to the assertion of the theorem we have a homogeneous relation in $C_{(n)}$ of the form 


$$
\sum_{i=0}^{k} f_{i}(u) \cdot u_{r}^{i}=0
$$

where $f_{i}(u)$ is a homogeneous polynomial on a finite number of elements from $O_{1} \cup \cdots \cup O_{n-1}$ but not involving $u_{r}$ which belongs to the same set, for $i=0,1, \cdots, k$. Further we have that $u_{r}$ is an element of highest degree occurring in (3.8.1) and that both $f_{0}(u)$ and $f_{k}(u)$ are not equal to zero. Apply the homomorphism $\Phi^{(s)}$ to (3.8.1). This gives that

$$
\sum_{i=0}^{k} f_{i}\left(\Phi^{(s)}(u)\right) \cdot\left(\Phi^{(s)}\left(u_{r}\right)\right)^{i}=0
$$

in $C_{s}$. Differentiate (3.8.2) partially with respect to $x_{j}$, which gives that

$$
\sum_{i=1}^{k}\left[\frac{\partial f_{i-1}}{\partial x_{j}}+i \cdot f_{i} \cdot \frac{\partial \Phi^{(s)}\left(u_{r}\right)}{\partial x_{j}}\right] \cdot\left(\Phi^{(s)}\left(u_{r}\right)\right)^{i-1}+\frac{\partial f_{k}}{\partial x_{j}} \cdot\left(\Phi^{(s)}\left(u_{r}\right)\right)^{k}=0
$$

Now by the induction hypothesis and Lemma 3.6, equation (3.8.3) gives that

$$
\frac{\partial f_{k}}{\partial x_{j}}=0 \text { and } \frac{\partial f_{i-1}}{\partial x_{j}}+i \cdot f_{i} \cdot \frac{\partial \Phi^{(s)}\left(u_{r}\right)}{\partial x_{j}}=0
$$

for $i=1,2, \cdots, k$, for all suffciently large values of $s$ and $j=1,2, \cdots, s$. Equation (3.8.4) implies that $f_{k}\left(\Phi^{(s)}(u)\right)$ is a nonzero scalar from $\Omega$ and

$$
k \cdot f_{k}\left(\Phi^{(s)}(u)\right) \cdot \Phi^{(s)}\left(u_{r}\right)=-f_{k-1}\left(\Phi^{(s)}(u)\right)
$$

for all sufficiently large values of $s$. By Lemma 3.6, this contradicts Construction 3.5 for the sets $O_{i}$, and thus gives the required results.

The nilpotent case is dealt with as $\dagger$ in $\S 2$.

THFoRem 3.9. Let $\overline{C^{k}}$ denote the closure of the $k$-th power ideal of ${ }_{1} C$ in $C$. Then $C /{ }_{1} \overline{C^{k}}$ is isomorphic to a subalgebra with unit element of a free $(k-1)$ th nilpotent associative commutative algebra with unit element over $\Omega$, provided that $\Omega$ is a field of characteristic zero.

Note 3.9.1. It can be shown that $C /{ }_{1} \bar{C}^{k}$ is isomorphic to $\lim \left(C_{n} / 1 C_{n}^{k}\right)$ under the homomorphisms of $C_{n} / 1 C_{n}^{k}$ onto $C_{n-1} / 1 C_{n-1}^{k}$ induced by ${ }^{-} \phi_{n}: C_{n} \rightarrow C_{n-1}$, for $\mathrm{n}=2,3, \cdots$.

\section{The interrelations between $L, A$ and $C$}

By Poincaré-Birkhoff-Witt Theorem, we have the following commutative diagram

$\dagger$ See also the proof of Theorem 3.8 . 


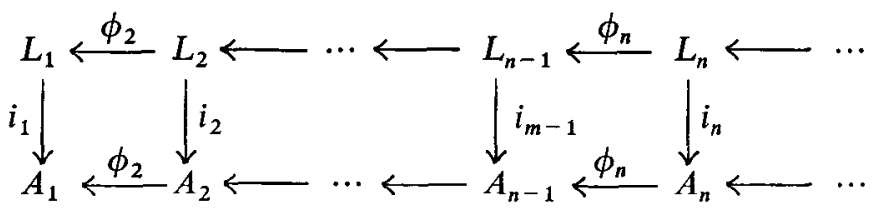

Further one has a Lie algebra monomorphism $i: L / A$. Hence if $\boldsymbol{L}^{e}$ denotes the enveloping algebra of $L$ and $\varepsilon$ the natural injection of $L$ into $L^{e}$, then one has an algebra homomorphism $\mu$ defined by the following commutative diagram

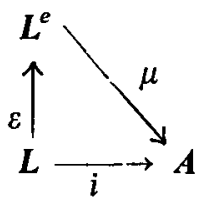

Note 4.2. The homomorphism $\mu$ defined by (4.1) is not onto when char $\Omega \neq 2$. In fact one can show that

$$
x=\left\langle x_{1} x_{2}+x_{3} x_{4}+\cdots+x_{2 n-1} x_{2 n}\right\rangle
$$

is an element of $\boldsymbol{A}$ which does not belong to $\mu\left(\boldsymbol{L}^{e}\right)$. This follows from Poincaré Birkhoff-Witt Theorem, reducing modulo ${ }_{3} A+i\left({ }_{2} L\right)$ (hence we are working in the algebra $\mathrm{C} /{ }_{3} \mathrm{C}$ as is shown in (4.8) and (4.9) below) and using an elementary argument concerning the rank of quadratic forms. For if $x$ belongs to $\mu\left(\boldsymbol{L}^{e}\right)$ then $\Phi^{(2 n)}(x)+\left({ }_{3} A+i\left({ }_{2} L\right)\right)$ has finite rank, which is bounded above by a number independent of $n$. However

$$
x_{1} x_{2}+x_{3} x_{4}+\cdots+x_{2 n-1} x_{2 n}+\left({ }_{3} A+i\left({ }_{2} L\right)\right)
$$

has rank $2 n$.

As one would hope one has the following

THEOREM 4.3. The algebra homomorphism $\mu$, which is given in (4.1), is a monomorphism.

Proof. Let $z_{\alpha}, \alpha \in M$ be a basis of the vector space $L$ over $\Omega$. Then, by Poincaré-Birkhoff-Witt Theorem, the set of all ascending products

$$
z_{\alpha_{1}} z_{\alpha_{2}} \cdots z_{\alpha_{k}}
$$

with $\alpha_{1} \leqq \alpha_{2} \leqq \cdots \leqq \alpha_{k}$ form a basis for the vector space $L^{e}$ over $\Omega$. Suppose that the element $z$ of $\boldsymbol{L}^{e}$ belongs to the kernel of $\mu$. Then $z$ can be expressed in terms of a finite number of the elements $z_{\alpha}$. This finite subset generates a free Lie algebra $B$ in $L$ over $\Omega$, by Theorem 1.7.1. Let $y_{y}, \gamma \in N$, be a set of free generators for $B$. Then $z$ belongs to $B^{e}$ and $B^{e}$ is a free associative algebra with unit element over $\Omega$ on the free generators $y_{y}, \gamma \in N$. Now we can write $z$ as a 
finite sum of the form

$$
\sum \lambda_{I} \cdot b_{I}\left(y_{\gamma_{1}}, y_{\gamma_{-}}, \cdots, y_{\gamma_{s}}\right),
$$

where every $\lambda_{I}$ belongs to $\Omega$ and $b_{I}\left(y_{y_{1}}, \cdots, y_{\gamma s}\right)$ denotes an ascending product of basic Lie monomials on the elements $y_{\gamma}, \gamma \in N$. This follows from Hall [2] and Poincaré-Birkhofi-Witt Theorem. Because of the usual identifications, we have that

$$
0=\mu(z)=\Sigma \lambda_{I} \cdot b_{I}\left(y_{\gamma_{1}}, \cdots, y_{\gamma s}\right)
$$

in $\boldsymbol{A}$. Apply the homomorphism $\Phi^{(p)}: \boldsymbol{A} \rightarrow A_{p}$ to this equation. This gives that

$$
\sum \lambda_{1}, b_{l}\left(\Phi^{(p)}\left(y_{\gamma_{1}}\right), \Phi^{(p)}\left(y_{\gamma_{2}}\right), \cdots, \Phi^{(p)}\left(y_{\gamma_{s}}\right)\right)=0 .
$$

This contradicts Lemma 1.6 (b) for sufficiently large values of $p$ if $z$ is nonzero.

We now look at the relationship between $\boldsymbol{A}$ and $\boldsymbol{C}$. We have the following commutative diagram

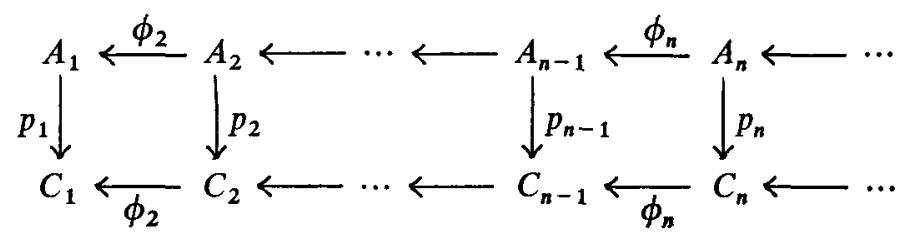

This defines an epimorphism $p: \boldsymbol{A} \rightarrow \boldsymbol{C}$. By Hall [2] and Poincaré-Birkhoff-Witt Theorem, we have that every element of $\operatorname{ker} p$ has a representation of the form

$$
\sum_{l}^{*} \sum_{I}^{*} \alpha_{I}(l) \cdot b_{I}(l),
$$

where every $\alpha_{I}(l)$ belongs to $\Omega$, the symbol $I$ denotes an ascending sequence $i_{1} \leqq i_{2} \leqq \cdots \leqq i_{k}$ of positive integers and $b_{I}(l)$ denotes the product $b_{i_{1}} b_{i_{1}} \cdots b_{i e}$ of basic Lie monomials on the elements $x_{1}, x_{2}, \cdots$ of total degree $l$ in $A^{*}$ and at least one of $b_{i_{1}}, b_{i_{2}}, \cdots, b_{i_{\mathrm{k}}}$ having degree greater than 1 . This is to hold for all terms of the sum.

\section{Concluding remarks}

We have not entered into the proofs of the corresponding results on the inverse limit of free nonassociative algebras over a field $\Omega$ to those proved in $\S 1$ for Lie algebras. The methods of proof are similar but technically simpler to those employed in the case of Lie algebras. Instead of using Theorem of Siršov [5] and Witt [7], one uses the simple Theorem of Witt [6].

Theorem 1.7.1, which states that $L$ is a locally free Lie algebra over $\Omega$ and the corresponding result for the free nonassociative case can be deduced from a much more general result. 
THEOREM 5.0. Let $\mathfrak{U}$ be a variety of $\Omega$-groups ${ }^{\dagger}$ such that the free algebras of this variety satisfy the following conditions:

(1) every free algebra of finite rank is hopfian, that is, is not isomorphic to a proper homomorphic image;

(2) the free algebras satisfy Schreier's condition, that is, every subalgebra of a free algebra is a free algebra (in $\mathfrak{U})$.

Then the inverse limit of an inverse system of locally free $\mathfrak{U}$-algebras and homomorphisms is a locally free $\mathfrak{U}$-algebra.

Proof. As in Higman [3], where this is proved for the class of all groups.

\section{References}

[1] L. Fuchs, Abelian groups (Hungarian Academy of Sciences, Budapest, 1958).

[2] M. Hall. 'A basis for free Lie rings and higher commutators in free groups', Proc. Amer. Math. Soc. 1 (1950), 575-581.

[3] G. Higman, 'On a problem of Takahasi', J. London Math. Soc. 28 (1953), 250-252.

[4] S. Moran, 'Unrestricted nilpotent products', Acta Math. 108 (1962), 61-88.

[5] A. I. Širšov, 'Subalgebras of free Lie algebras', Mat. Sb. 33 (75) (1953), 441-452.

[6] E. Witt, 'U'ber freie Ringe und ihre Unterringe', Math. Zeit. 58 (1953), 113-114.

[7] E. Witt, 'Die Unterringe der freien Lieschen Ringe', Math. Zeit. 64 (1956), 195-216.

The University

Canterbury, Kent

England

The University

Papua and New Guinea

† Groups with multiple operators in the sense of P. J. Higgins. 